\title{
Unweaving the human response in daylighting design
}

\author{
Prof. Marilyne Andersen* \\ Interdisciplinary Laboratory of Performance-Integrated Design (LIPID), School of Architecture, Civil and \\ Environmental Engineering (ENAC), Ecole Polytechnique Fédérale de Lausanne (EPFL)
}

*Corresponding author: EPFL-ENAC-IA-LIPID, Building GC A2 401, 1015 Lausanne, Switzerland. marilyne.andersen@epfl.ch

\begin{abstract}
Daylighting as a research topic situates itself at the interface between psycho-physiological and environmental factors, bringing together questions relevant to architectural design and building engineering, but also to human physiology and behaviour. While daylighting has a strong impact on human health and well-being, and an undeniable association with (subjective) emotional delight and perceived quality of a space, it is also highly dynamic and variable in nature, based on a combination of predictable (sun course) and stochastic (weather) patterns. This makes it both a challenging and essential aspect of how "performative" a space can be considered.

This paper aims to discuss selected research developments regarding how architectural engineering and other domains of science could be more strongly bridged to address the need for meaningful decision support in daylighting design: how can we better integrate the complexity of human needs in buildings into effective design strategies for daylit spaces? As a basis for discussion and to illustrate this overview, it describes a unified goal-based approach in an attempt to address the multiplicity of perspectives from which daylighting performance can - and should - be evaluated in building design. Through five very different perspectives ranging from task-driven illumination or comfort to human-driven health and perception, it proposes a simulation and visualization framework in which one can start approaching these from an integrated approach.
\end{abstract}

Keywords: daylighting, climate-based modelling, goal-based, performance metrics, non-visual lighting, design decision support

\section{Introduction}

The ultimate impact buildings have on our lives is fundamental: buildings represent a major part of our life, with a share of over $40 \%$ of overall energy use, waste and $\mathrm{CO} 2$ emissions [1], almost $50 \%$ of the population now living in cities, and $90 \%$ of time typically spent indoors. With lighting, heating and cooling being the three most energy-demanding building functions [1], it appears very clearly how efficient daylighting and solar control strategies can have a tremendous impact on energy use [2]. But any savings can only be effective if one also carefully accounts for our comfort, well-being and health criteria.

The integration of building performance criteria into the design process has received a great level of attention in the last two decades. Simulation models of increasing degree of sophistication [3] have been developed and a wide range of metrics and of tools are now available and still being developed to support our search for minimizing energy consumption or ecological footprint. One of the underlying principles for this search is that the performance of a space will typically increase with user satisfaction and decrease with energy consumption [4], the latter being used towards discomfort compensation until occupant satisfaction is 
reached. In today's energy crisis, minimizing this compensation becomes essential but requires a very good understanding of our comfort requirements, used as triggers for (predicted) action on our environment according to still poorly understood interaction patterns with building controls (dimming, thermostats, shading etc) [5-10]. In the contemporary context of building for sustainability, we must move beyond energy-centric approaches and consider sustainable design from a broad perspective, focusing also - or actually starting with - quality of life, occupant satisfaction and psycho-physiological human well-being.

Static comfort thresholds can only provide an incomplete picture and have led, in the 30ies to 60ies, to an era of windowless, air-conditioned offices and classrooms as ultimate symbols of progress specifically because they adhered - under full control - to accepted standards for comfort. We have now realized that our needs far surpass these universal ones [11-13] and include a longing to bond with our living environment (biophilia hypothesis) as well as a need to stay connected to its ever-changing nature through windows and views [14].

While we share fundamental needs driven by evolution, it is very clear that people value things differently, and have needs far from being uniform at several levels (Figure 1):

- diversity: Individual preferences about privacy, temperature swings, or architectural character e.g. depend on factors ranging from cultural background and social status to health and age.

- variability: The temporal variability of our needs results from our inherent attachment - at both the psychological and physiological levels - to a very variable outside environment (seasons, time of day, weather, vegetation, light-dark cycles).

- boundaries: In today's urge to design sustainably, we must

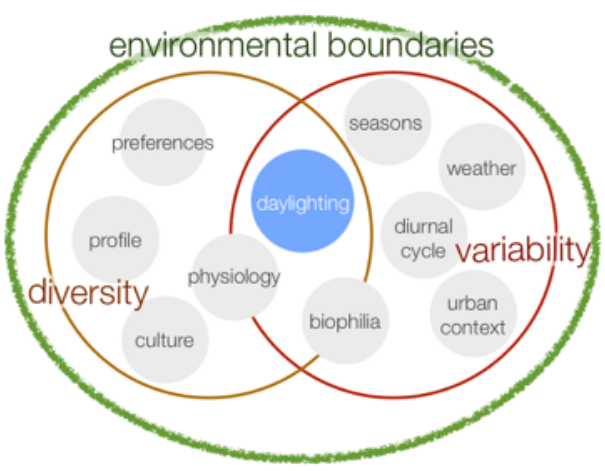

Fig. 1: Ingredients of human-driven design think within an overarching reality of finite resources; to reduce our carbon footprint and use of non-renewable energy, the building sector - that accounts for a $40 \%$ share of these resources - plays a pivotal role.

The architect is thus faced with multiple, highly variable, bounded criteria that can conflict but need to be brought together to lead to a satisfying solution. Yet unlike clothing that can easily and continuously be adjusted to both user preferences and outside conditions, buildings are constrained to a somewhat limited set of materials and to basic adaptation (shading, operable windows), which prevents them from responding to individuals to the same degree of refinement as clothing: preferences have somehow to be synthesized, and the main role of the architect is to do this synthesis. On the other hand, the ultimate balance between all factors and constraints leading to a satisfying design solution cannot be solely based on measurable criteria: a design process does not reproduce conventional optimization because of its inherent non-linearity and reliance on elements that cannot always be objectified.

This paper investigates a selection of research perspectives aiming to reinforce links between building science and architecture through trans-disciplinary approaches reaching out to other fields such as photobiology and psychophysics regarding perceptual, visual (comfort-based) and non-visual (health-based) daylighting design. It proposes a goal-based strategy to address design decision support, and discusses five interpretations of "good daylighting" performance objectives, brought together in a unified visualization framework for daylighting simulation centered around human needs. 


\section{Dynamic daylighting for variable and diverse human occupants}

Although basic physiological needs can be considered similar enough for most humans, leading to somewhat predictable ranges of acceptability regarding comfort and well-being in buildings, some degree of diversity and variability is inevitable regarding building occupants' preferences and perceived needs, particularly marked as they relate to daylighting penetration [5].

As far as illumination is concerned, these needs typically get translated into target illuminance values or ratios [4,6,15-16] - with the earliest "daylight factor" recommendations dating back to the end of the 19th century. Established metrics relevant to daylight penetration have indeed been focusing on finding benchmarks for task illuminance and visual comfort (glare avoidance) $[4,6-7,15]$ yet with varying degrees of applicability beyond the conditions in which they were measured, and with results that are often difficult to compare [6]. More recently, climate-based modelling has become a widespread approach - at least in research but also to some extent in practice - so as to consider daylighting on an annual basis [4,10,17-20]. Other studies have also looked at individual preferences [5], or at "light quality" indicators typically derived from luminance averages or ratios [21]. Different daylight metrics concepts have been proposed, such as associated to entire space areas [18] or viewed scenes [22] rather than individual detection points, and based on relative approaches for more complex systems [23]. The latter helped to shift the focus back on daylight variability, not only its spatial distribution [18-19,22].

How well a given space is daylit is by essence a multifaceted question: it is a key factor in how well any visual task will be performed, a main driver of occupant satisfaction regarding visual and thermal comfort (and hence energy consumption resulting from trying to meet comfort requirements), has a strong impact on human health and well-being, a close association with the perceptual quality of a space, and is highly dynamic in nature as a result of daily and seasonal variations. .

The multiplicity and variability of our needs regarding (day)light exposure have thus clearly been a topic of investigation for years. To more deeply embed the diversity and variability of human needs as foundational elements of daylighting design and put human occupants back at the core of the question, we now also need to reach out to other research fields, so as to bring new insights and a deeper understanding of how we interact with our environment.

This requires first to uncover - unweave - essential relationships between human occupants and a daylit environment with a focus on their dynamics, and to establish cross-disciplinary bridging with research fields a priori remote from architecture, such as photobiology and psychophysics (Figure 2). We propose to start by challenging the notion of human well-being in a daylit space at three inherently dynamic levels of humanspace interactions that have not yet been consistently integrated as design guiding factors, yet are fundamental to how a space is actually experienced:

- as a human inhabitant of a living space who needs to be in an environment conducive to health

- as a user of a (work)space who performs a task

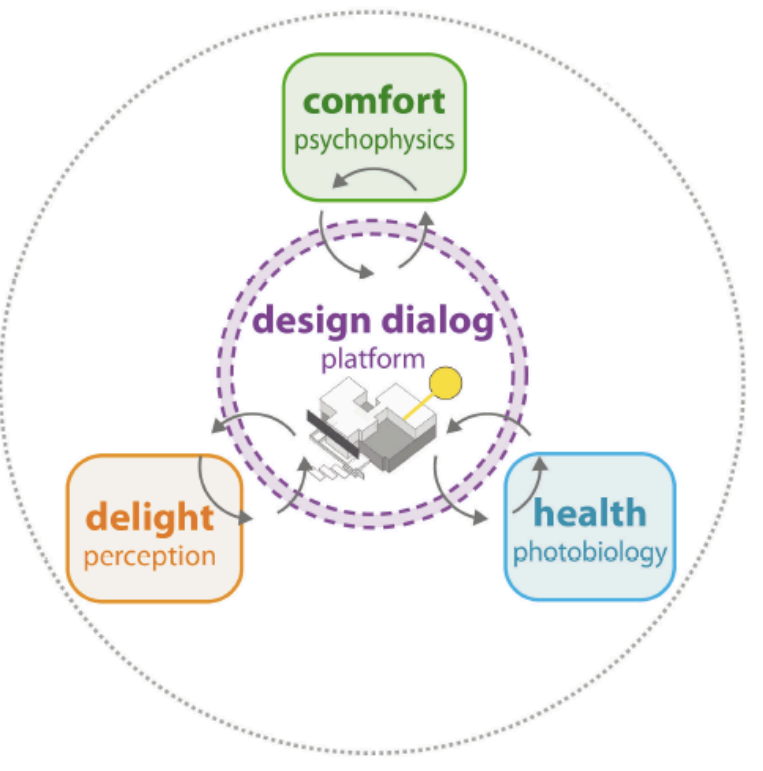

Fig. 2: Inter-related aspects of human-responsive (day)lighting design reaching out to other fields of research 
yet behaves dynamically in a space

- as a witness of a delightful space who wants to enjoy it.

\subsection{Healthy lighting}

Humans need to be in an environment conducive to health and have physiological light exposure needs, whose time- and spectrum-dependent non-visual effects we only start to understand thanks to findings in circadian photoreception research [24]. A new research field in non-image forming lighting has indeed emerged in photobiology from the recent discovery of a specialized photoreceptor in our eye's ganglion cells - containing a light-sensitive pigment named melanopsin -, responsible for synchronizing our internal circadian pacemaker, making light an essential element of healthy living.

\subsubsection{Light as a therapeutic tool}

Amongst the environmental factors that strongly influence our sense of well-being and ultimately our health, light plays an essential role as the primary environmental time cue for synchronizing the internal circadian clock. Failure to receive this information can result in non-24-hour sleep-wake disorder [25], the most common being jet-lag and shift-work. This condition can lead to insomnia, excessive sleepiness, and increased risk of diabetes and some types of cancer [26]. On the other hand, light has revealed itself as an effective therapeutic tool against seasonal affective disorder (SAD), while under real-world conditions, exposure to robust light-dark cycles was shown to be associated with better workplace performance [27] and better patient outcome in hospitals [28].

In addition to stimulating visual responses, light can actually induce non-visual responses through two types of effects: through phase shifting effects on the circadian clock, and through direct activating effects [29-30]. These effects are mediated primarily via the novel non-rod, non-cone photoreceptor melanopsin, which is most sensitive to blue light and exhibits a different sensitivity to radiant flux than the pigments in rods and cones [24,31]. The discovery of this novel photoreceptor, the intrinsically photosensitive retinal ganglion cell (ipRGC), has led to consideration of the non-visual effects of light as an important element of healthy lighting design in addition to vision [32-33].

Researchers have identified five main characteristics of light exposure for melanopsin stimulation: intensity, spectrum, duration/pattern, history, and timing [24,34] with desired properties that so far seem - quite unsurprisingly as we humans evolved under it - to be dominant in daylight. The 24-hour light-dark cycle resets our circadian clock, which in turn synchronizes much of our physiological processes and social behavior. Beside these circadian resetting effects of light, bright light can directly suppress melatonin and increase alertness, when contrasted with dim light conditions [29-30,35-36]. It has been shown that brief exposure to bright light may have a greater impact on the circadian clock than was previously recognized [37-39]. Prior exposure to bright light can reduce the amount of non-visual responses [40-41]. Furthermore, blue light was demonstrated to be more effective in enhancing alerting effects compared to green light [42], highlighting the unsuitability of illuminance to evaluate the non-visual effects of light.

In a recent field study, the effects of exposure to blue-enriched white light during daytime work hours were investigated in comparison to white light [27]. Blue-enriched white light improved subjective alertness, performance, mood, and sleep quality. This suggests that blue-enriched white light in real-world settings can have beneficial effects for people working normal office hours, which makes it an appealing alternative to enhance alertness. Another study, where subjects were exposed to two realistic office lighting conditions for 
two consecutive days, reported higher subjective alertness in the afternoon in response to prior bright daylighting condition ( 1000 lux) compared to typical artificial lighting condition ( 180 lux) [43].

A spectral sensitivity function for the human non-visual system has not yet been standardized, primarily because the maximum sensitivity for the non-visual system is not definitive, falling between 446 and $483 \mathrm{~nm}$ for melatonin suppression [44]. Moreover, the photoreceptors' contribution to non-visual responses may differ with intensity and duration of a light exposure [45]. It seems that the non-visual responses to light like melatonin suppression, phase shifting, alertness, etc. cannot be predicted using a single spectral sensitivity function like the $\mathrm{V}(\lambda)$ curve for photopic vision [46]. The right balance between optimizing light simultaneously for visual and non-visual functions has not yet been found, nor do we know how to model the non-visual light responses, especially during the day. The vast majority of studies - mostly using monochromatic light or stationary electric lighting - have been conducted at night or in dim-light conditions and been focusing on non-visual effects at the onset of light exposure [47]. Only small pilot studies have started to address daytime exposure [48] though studies did show that bright light could increase subjective alertness during the day as well [35-36].

These recent findings have brought the non-visual effects of light to receive increased attention among researchers in the lighting community $[47,49]$. Though a number of models have been developed to predict the effect of different sleep-wake schedules on human performance and alertness [50], no mathematical models currently exist that can predict the direct non-visual effects of light as a function of intensity, spectrum, duration, history, and timing of exposure, which bears the risk of making decisions based on an incomplete understanding of the effects.

\subsubsection{Non-visual lighting as a step towards healthier building environments}

Is it possible to predict if architectural spaces are good for health and wellbeing using computational models, thereby supporting the design of healthier building environments?

Before we can answer this question, it is necessary first to understand the dynamic relationship between light and human non-visual responses. Modeling can be a useful tool not only to gain deeper understanding of complex systems but also to point out gaps of knowledge, suggest specific experiments and serve as a means to study practical implications of simulated human responses. One challenging aspect is the fact that the non-visual system adapts its responses to changes in light intensity and spectral composition over a much longer timeframe than the visual system $[37,45]$.

Very few studies can be found that use this new knowledge to investigate which design factors can increase the non-visual potential of architectural spaces. In the very first modelling frameworks that were proposed to address this question [19,33,51-52], a static approach was chosen in the sense that the slower adaptation of the non-visual system was not taken into account, nor were spectral sensitivity shifts and desensitization effects after prolonged exposure included. While there does not appear to be sufficient information on nonvisual responses to light to produce a detailed mathematical model that would work in real-world settings, experimental findings still offer the means to advance and validate novel design support tools to assess how architectural spaces might affect human health and wellbeing, and efforts in this direction are underway to model direct non-visual effects as a function of exposure duration and pattern (including timing and history), spectrum and intensity [19,53-55], as illustrated in Figure 3.

Fig. 3: Prospective non-visual light-response model 


\subsection{Comfort dynamics}

Users of a workspace usually need to perform tasks for which comfortable visual conditions are needed, and lighting has been acknowledged many times as a key factor in workplace satisfaction and ergonomics.

\subsubsection{Measures of visual comfort}

In terms of visual comfort, there is a general consensus that glare is the main cause of occupant interactions with shading and thus a major source of potential dissatisfaction from occupants [5,8]. The more common type of glare for interior spaces, referred to as discomfort glare, induces negative reactions that are critical to be solved with proper design and lighting controls [10,56], though no observable disability [57]. Despite numerous efforts in coming up with glare indices through surveys conducted either with luminaires [15,57-58] or, more recently, with daylight [7,59], predicting occupant discomfort with a glare index still poses important challenges in design, and recent studies have started to consider time of day, as well as other temporal and personal factors, as parameters to be possibly taken into account in the subjective evaluation or prediction of glare sensation $[6,10]$.

There are at least 7 recognized indices for glare [7,15,59], and all are based exclusively on subjective assessments (self-rated comfort). One index of note is the Daylight Glare Probability (DGP) [7], based on sidelit office conditions and considered the most reliable index for daylit workspaces, as it is the only one actually derived from daylighting conditions. While there are many situations where these different indices disagree with each other partly because of their unequal accuracy for electric and daylighting sources [6], most are drawn upon the same four physical quantities: luminance, size and position index of the glare source, and the general field of luminance the eye adapts to [10]. The position index expresses the change in discomfort based on the angular displacement of the glare source from the line of sight $[58,60]$.

A major limitation shared by all known glare indices, however, is to ignore where we actually look and what causes a view pattern: the assumption is that the view direction is fixed [60], leading to glare evaluation models that consider a single view towards the task (most common approach), or a pre-defined range of views to account for probable head and eye movements, such as in the recently proposed 'adaptive glare' concept [61]. Actual changes in view direction will have a strong impact on the assumed position of the glare source and indirect implications on glare adaptation or angular size, thus ultimately a significant influence on glare evaluation outcomes: if lighting and perceived comfort in turn influence the preferred line of sight, this influence should also be reflected in the glare index and head and gaze dynamics, that psychophysics can help us better recognize, should be embedded in it.

\subsubsection{Outreach to vision research and eye-tracking technology}

While early studies already pointed out that visual context, task and expertise all play a key role in driving eye movements [62], we now know that they can override each other depending on the specific conditions [63-64]. Changes in the line of sight may serve to scan the visual environment or to stabilize gaze (volitional or reflexive movements), while rapid shifts in eye position - known as 'saccades' - constitute a prominent class of eye movements in humans [65]. Computational models that aim at predicting gaze orientation typically focus on the control of large eye movements by low-level stimulus features, such as luminance contrast, color, orientation or motion [66]. Experimentally, however, gaze is long since known to be influenced by task [62], context [64] or economic value [67]. Computational models that include such "top-down" factors typically focus on visual search [67], which is of high theoretical interest, but rare in real life. Other 
approaches to eye movements in realistic tasks either used very reduced stimuli or restrained settings, such as driving [68], food preparation [69], or sports [70]. For less constrained scenarios, laboratory data has very limited predictive power for the real-life situation [71]. Although different eye movement classes coexist and interact with each other, up to recently studies of eye movements during natural perception have mainly focused on saccades with constrained head and body. Surprisingly few studies can be found on the relationship between eye movements and building-induced visual context, such as a window [72], and none of these linked eye movements to comfort perception until very recently [73-75].

Eye movements as a variable for visual comfort studies can actually not be addressed with psychophysical procedures typically used for glare evaluation: the latter rely on subjective occupant perception whereas people are oblivious to their specific head and eye movements. From previous work demonstrating the profound influence that cognitive factors have on eye movements [63], one can hypothesize that there are clear relations between gaze patterns, glare and luminous environment; our preliminary investigations to test this hypothesis [73-74] demonstrated the potential of wearable eye-tracking technology [76] to reveal such relationships. As conceptually illustrated in Figure 4, discomfort glare models could therefore benefit immensely from integrating line of sight dynamics together with daylight dynamics, while also pushing the boundaries of gaze modeling beyond local stimulus-driven saliency. Uncovering these relations has the potential to change our current approach to glare prediction and comfort in workspaces and open up new research pathways on how a space is experienced visually (i.e. what attracts our eye), where investigations have remained limited to search for main preference trends.

dynamic comfort

glare - gaze allocation relationships head/gaze dynamics

eye-tracking

inter-dependencies

Fig. 4: Integration of view direction in visual comfort models

\subsection{Delightful spaces}

Architects understand the importance of natural light also in its ability to generate an atmosphere, reveal design, draw a choreography of geometry and light, provide texture to built volumes or add dynamics to a space - in other words, to enhance visual interest and provide emotional and experiential delight to the witness seeking to enjoy it.

The perception of daylight within architectural space is unquestionably an important aspect of visual performance and impacts the ways in which that space is experienced. Contrast draws our attention toward spatial complexity and highlights areas of material transition, while the dynamic nature of sunlight generates varied luminous effects over time [77]. The visual effects of daylight are subjectively perceived and evaluated by each occupant, making it very difficult to enforce them as design factors even though they may ultimately drive many design decisions. The perceived qualitative aspects of daylight in a varying indoor space are underserved by the metrics currently available to designers: architecture must 'perform' in both qualitative and quantitative criteria yet architects still have to rely on intuition and experience to evaluate their dynamic effects against their intended programmatic use. We must work to re-establish the role of emotional and perceptual indicators in our language about performance.

While some metrics have emerged to try and quantify 'light quality' through identifying a relationship between brightness, contrast and occupant preference [78-80], they are generally based on occupant surveys of existing scenes or static digital images of an interior space taken at key incremental moments. Although occupant surveys were once the primary method of data collection, digital photographs have become a useful alternative for practical purposes [78]. Since the advent of HDR imaging, we are now able to produce 
digital photographs and renderings with a broader range of luminance data that more accurately capture a scene from an occupant's point-of-view [21].

Two dimensions that are widely accepted to impact the field-of-view are average luminance and luminance diversity [78]. The former has been directly associated with perceived lightness and the latter with visual interest. Those metrics that take advantage of HDR digital images aim to correlate factors such as view size, average luminance, or luminance diversity with an occupant's perception of pleasantness, excitement, and spaciousness of the view as established by surveys $[21,78]$. The studies that focus on average luminance are generally associated with perceived lightness, while luminance diversity (typically $\mathrm{min} / \mathrm{max}$ ratios) is often associated with visual interest. Other studies have used genetic algorithms to predict occupant preferences toward average luminance and uniformity within a specific program environment, such as an office environment [21]. Although these findings are somewhat consistent in pointing out that occupants seem to prefer bright, non-uniformly lit environments with some luminance diversity [79-80], none of these studies addressed the question of dynamic variability of daylight as it is impacted by seasonal and daily variations in strength, climate, and solar orientation until the recently proposed spatial contrast and variability concepts $[19,22,81]$ summarized in Figure 5. Outside of the Luminance Difference Index [80], and unlike the human brain that is capable of discerning such effects by mere observation [82], there is also a lack of metrics that can distinguish between compositional variations in contrast across our-fieldof-view, so as to move beyond the early findings from $[19,22,81]$ to describe the experiential impacts of architecture.

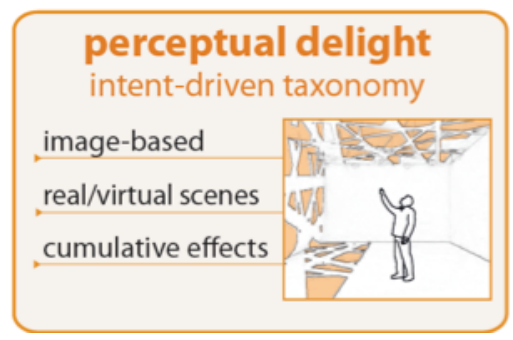

Fig. 5: Towards rendering-based perceptual daylight analysis

\section{Decision support for human designers}

Although tools are typically developed based on well-defined computational processes, the latter are less easily compatible with the non-deterministic, ill-defined and unpredictable nature of the design process [83], which is exactly where its creativity lies. To get technology to support human-centered design, we must put designers back into their essential role, which is not to test and optimize - tasks for which a computer might be more efficient indeed - but to know what to look for.

\subsection{From conflicting needs to an integrated built form}

How can we help architects synthesize complex, dynamic and often conflicting goals into an integrated built form? State of the art approaches seem to consistently aim at translating ill-defined problems into wellstructured ones [84-85] - though in various ways - so as to be able to rank [86-87], guide towards [85,88-90] or generate [91-93] design solutions using objective analysis or optimization methods. But to effectively support decision making, such methods must also respect - or even fuel - the inherently creative and unpredictable design exploration process.

Amongst the notable attempts to improve quality, speed or convergence of the ever growing number of optimization and computer-aided design (CAD) methods in architecture, we find knowledge-bases [94] amongst which some relevant to lighting [95-96], but only very few [87] applicable to climate-based daylight modelling. We also find meta-modeling [97], multi-objective approaches [98], and any combination thereof such as in building energy modeling. Despite the breadth of such methods, the basic, conventional approach tends to remain sequential (Figure 6): design decisions are explored to match pre-specified criteria through 
repeated evaluations of a numerical model [99].

Yet in a design process, the aim is not to find an optimal solution but to produce a range of satisfactory ones, with different priorities and compromises [85]. The recently introduced performative design paradigm [100] provides an interesting framework trying to address this issue: it reverses the traditional design process in which generation of form is prioritized over performance evaluation and uses desired performance as a mechanism to digitally generate and modify the design. Due to the very nature of architectural design, however, the problem must remain ill-defined in a number of respects, as no criteria exist that can test a given solution, nor is there any systematic process in which such criteria could be applied. The problem space is by essence incomplete and undefined because its very definition would require the architect to explicit all decision criteria a priori [84], something (s)he cannot - and maybe should not - be asked to do. Moreover, at each design iteration, the goals, constraints and problem-space might change, while qualitative aspects - such as architectural aesthetics i.e. very hard to integrate into a formalized optimization process - will further increase the ill-definition of the problem.

Attempts at such an integration have been proposed through multi-objective

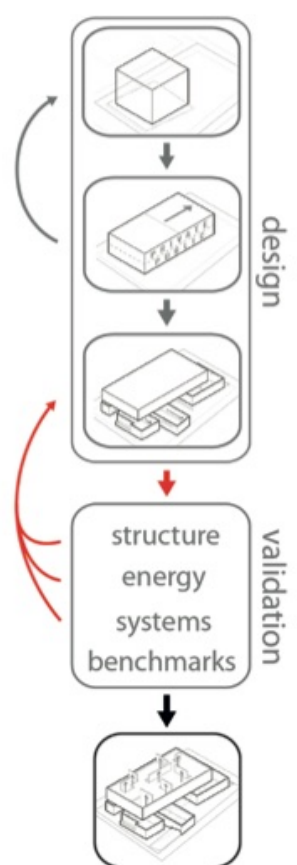

Fig. 6: Sequential design process towards improved performance optimization using Genetic Algorithms (GAs) [91,101]. While a significant body of knowledge has already been produced in the area of generative design [92] - though, again, with very few papers addressing daylighting $[86,91,93,102]$ - these methods remain limited in the level of interactivity they allow during the decision making process. They also produce solutions based solely on performance criteria, not on an understanding of design: fast convergence and satisfying results can be achieved through this approach (e.g. for floor layout design [101]), but the problem still needs to be split into a set of smaller, well-defined problems [84], which greatly impedes creativity and innovation potential.

An alternative approach is to include the user in the optimization process through different existing techniques. Interactive optimization is one, such as for "User hints" frameworks that insert domain knowledge to avoid local minima [103]; we also find expert systems [85,88,104] -, or human-guided search [105]. Other well-known approaches include Pareto-space optimization (pre-calculated Pareto front from which solutions can be selected [106]), interactive evolutionary computation (IEC) that emerge from interactive evolution techniques [107], or Mixed-Initiative Systems (MIS) with added visualization features [108]. Such optimization approaches, that engage the user in the process, unfortunately all share the same important limitations: they require a well-defined problem (sub)space, they do not offer the user any control on the "black-box" computation of solutions which are, as a result, unlikely to respect his/her design intent, and they will not find sub-optimal alternatives from a performance standpoint that might be preferred by the user for other reasons. Additional issues relate to user fatigue: most of these methods require up to or beyond a hundred user inputs, leading to a decrease in performance [107]. This problem is even more acute for early stage design given the limited time dedicated to energy or daylighting performance optimization although it is exactly during those early phases that crucial decisions regarding ultimate performance are taken. This can be partly solved by resorting to an iterative approach [88] where recommended design changes are presented step-by-step to increase educational value, and can be skipped to increase choice freedom, all highly desirable features as revealed by the user studies that were subsequently conducted [90].

While these outcomes are promising as a basis to build upon, we still need a breakthrough to fully embed 
the ill-definition of design into a dynamic, human-responsive computational framework. Future developments could for instance build upon findings in human-computer interaction that demonstrated the potential of virtual experts in improving teaching quality [109] and the effectiveness and attractiveness of a conversational (dialog-based) type of learning [110] for tackling ill-defined problems [111].

\subsection{A goal-based approach to support decision-making}

Architects are increasingly using digital tools during the design process, particularly as they approach complex problems such as designing for successful daylighting performance to either evaluate task performance through workplane illuminance calculation [17], energy impacts of daylight such as active electric lighting, heating or cooling needs to compensate for excessive or insufficient daylight [1-3], or its impact on comfort and in particular on glare-based sources of discomfort within the visual field [10].

What these numerous tools and approaches have in common is the aim of trying to either define or meet broadly acceptable (yet sometimes population or condition-specific) target values so as to guide design towards objectively "better" performance. Yet daylighting is known to be a field where strictly defined numerical boundaries are not enforced. There is a vast range of parameters and values that contribute to "good" daylighting design and which make absolute performance targets of questionable relevance: the question of "how good is good?" is indeed far from trivial with the multifaceted, highly variable nature of daylighting performance, about which people - occupants as much as designers - have highly diverging opinions.

The 'human' challenge at hand is two-fold. It comes from the human nature of the designer, which remains the main driver of a design process: the ultimate balance between multiple, often-conflicting criteria cannot solely be based on measurable parameters, thus the design process must remain non-deterministic. And it comes from the human nature of the occupants, which encompasses individual diversity and temporal variability: as we know, to feel comfortable in a daylit space can result in very different constraints depending on the time of day, the season and the location of the building, and human factors will induce diverging preferences for comfort from individual to another. The necessary flexibility and dynamic response of design goals also applies to our cyclic physiological needs or to the ever-changing ambiance of a space that contributes so intimately to its uniqueness.

The ultimate objective is to provide building designers with the means necessary to assess critical parameters in a successful design and efficiently combine qualitative and quantitative criteria in the solution search process. With daylighting in particular, performance must be measured against goals that vary over time, by occupant profile and/or be driven by the designer's intent, which necessarily retains a part of subjectivity. What we must identify is how a building should respond to two inputs: on the one hand to what we have, i.e. analysing the resources available to work with (i.e. the building's environment whether natural and/or built, its localisation, climate etc); on the other hand to what we need, to determine whether and how the needs of the building's occupants can be met.

Where technology can help is in allowing the architect to go beyond his or her own sensitivity: the extent to which a design answers even dynamic and highly refined goals can probably be determined systematically though it might require enormous calculation capacities - but the objective should remain to help the architect extract an informed synthesis. This process should in principle start by defining where one wants to go in some form: any design option or material choice, for instance, is - consciously or unconsciously gauged against a reference enabling us to make a decision and move forward. How to make this reference 
explicit or objective is sometimes where the challenge lies. If one can express this reference as a performance goal - which can then be gauged against more or less refined models or metrics - simulation can offer a very powerful support for decision-making [90-91].

\subsection{Five declinations of "good daylighting" goals}

Preliminary concepts have already been investigated to take a goal-based approach on daylight performance, amongst which the following five perspectives were proposed with the explicit intent to move towards a unified framework of decision support in the design process regarding daylight performance [112]:

1) Workplane illuminance (visual task performance), based on the concept of Acceptable Illuminance Extent (AIE) introduced in [18];

2) Discomfort glare (visual comfort), based on the concept of Glare Avoidance Extent introduced in (GAE) [18] but applied to full Daylight Glare Probability (DGP) [7] calculations;

3) Solar gains management, based on the Solar Heat Scarcity / Surplus (SHS) concept introduced in [18];

4) Non-visual effects (direct and circadian), based on a dynamic model focusing on time-dependencies of spectral response and light exposure adaptation, including prior history of light exposure [19,53,55];

5) Perceptual daylight, based on the combined occurrences of contrast and variability within viewed scenes $[19,22,81]$.

Many other approaches can be found that try to address each of these perspectives individually; it is together that the five perspectives considered here offer an interesting potential to support a consistent approach towards "good daylighting" based on user-defined goals. As illustrated in Figure 7, the underlying focus pertaining to all five perspectives is the combined temporal and spatial dynamics of daylighting within a space. Whereas each one relates to a specific attribute or impact of daylighting, the aim is to be able to translate the latter into objectifiable - though not necessarily explicitly numerical - goals.

The five perspectives are briefly presented here, together with their associated "metrics"; more details about the latter can be found in the previously published papers (referred to below) in which they were introduced.

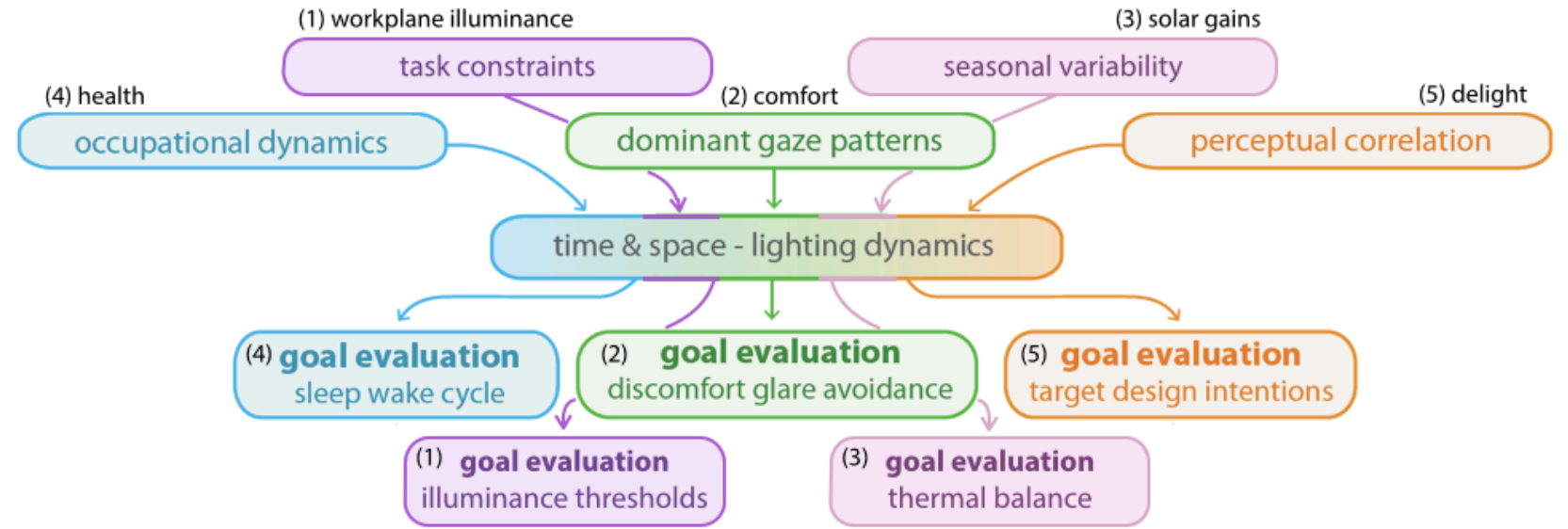

Fig. 7: Five perspectives regarding daylight performance brought together around a goal-driven approach emphasizing spatial and temporal dynamics

\subsubsection{Visual task lighting}

The illuminance-derived metric considered in the present framework, called Acceptable Illuminance Extent (AIE), has been introduced in [18] and calculates how the percentage of a user-defined area of interest in 
which the illuminance stays within a chosen range varies over time. It therefore simply relies on surface illuminance (workplane, wall etc) and is typically double-bounded (lower and upper bounds) although upper bounds only make sense when too much light is an issue (such as for artwork exhibits e.g.).

This metric requires one or more light receiving surface(s) (or perimeter(s)) to be freely defined by the user in a 3D model, and the boundary conditions to be chosen. The latter should answer the following questions:

- below what illuminance threshold in [lux] shall we consider that there is basically no useful daylight penetration (acceptable low) and above what threshold is illuminance perfectly satisfactory (desired-low)?

- above what threshold (if any upper bound is necessary) are the conditions starting to be less ideal (desired-high) and above what threshold is there definitely too much light (acceptable-high)?

This concept is illustrated on the first row of Figure 8 below.

\subsubsection{Comfortable lighting}

The metric called Glare Avoidance Extent (GAE), also introduced in [18] and used in the present context, relies on the Daylight Glare Probability or DGP [7] (and its established boundary conditions) in combination with a principle similar to the AIE in the sense that it conveys an evaluation of how "glary" a whole zone within a space is by evaluating the percentage of glare "sensors" (automatically generated so as to populate the perimeter of interest with a well-distributed set of view locations and directions) that show intolerable or disturbing visual conditions.

This metric requires the user to either define a perimeter of interest (portion of a space) in which he/she is interested to assess glare from arbitrary view locations and directions (to assess the overall glare risk of the area) or to define one (or a set of) specific view location(s) and direction(s) that are critical in this particular space, where a conventional DGP analysis should be conducted. As illustrated on the second row of Figure 8 , the user-defined goals thus pertain to how sensitive to glare the considered space (or space portion) is to glare, which determines which DGP thresholds to apply [7].

Future developments should add a new dimension to visual comfort analysis by including view direction patterns so as to be realistic about how a workspace is actually experienced. Building upon recent advances in eye-tracking methods from psychophysics, research is currently being conducted to integrate objective inter-dependencies between gaze patterns and visual context in a dynamic glare model [73-75], so as to create a robust and more realistic predictor of perceived comfort that accounts for gaze dynamics and, ultimately, help promoting productive environments.

\subsubsection{Seasonal lighting}

The energy impacts of satisfying thermal comfort benchmarks with active heating or cooling can become significant if excessive or insufficient daylight and its associated tradeoffs - namely solar gains or excessive heat losses due to large areas of glazing - must be compensated for to a too large extent. To account for both overheating risks and potentially reduced heating loads as a function of season (and building type, function, occupation etc), a new solar gains metric called Solar Heat Scarcity / Surplus (SHS), again introduced in [18] and illustrated on the third row of Figure 8 , is used here to convey the urgency of either allowing more direct solar gain or avoiding it, based on revisited balance point calculations.

The calculation of SHS requires material information beyond the geometry and surface reflectivity inputs that are typically needed for the other metrics, in particular regarding heat transfer properties of the envelope components. In addition, estimated values for heat gain, ventilation, occupancy, and operational information 
will be required, based on building type, as well as location (hence, climate type and weather statistics).

\subsubsection{Healthy lighting}

As architecture mediates the boundary between the external environment and the human body, it becomes the most impactful element in providing building occupants access to natural light. Yet no model currently exists to predict the direct effects of light exposure on human health and well-being. Efforts are currently being put on building a computational model to simulate non-visual responses from discrete intensity and spectral inputs $[19,53,55]$. This work will build upon a unique bridging between neuroscience and architecture that was initially proposed in an early non-visual lighting simulation framework for architectural design with a focus on healthcare design [33], using illuminance thresholds derived from [29] and assuming neutral interior surfaces and a time-independent spectral sensitivity curve. This study was later extended to include the impact of view location and direction in housing [51] and a differentiation based on day periods of light exposure [52]. Despite the immaturity of this research, it seems possible to start building models which can describe the complex temporal and spectral inter-dependencies of the non-visual responses during both day and night, and that embeds the flexibility necessary to incorporate new findings as photobiology research advances.

The objective is to integrate time- and spectral-dependencies of non-visual responses to light, and reconsider light intensity thresholds as a function of prior history of light exposure. Practically, this means taking the intensity, spectrum, duration/pattern, history, and timing of light exposure as variables to evaluate non-visual responses to light (i.e. affecting health) as a dynamic mathematical model of the non-visual system, including space-related color effects and behavior scenarios based on occupational dynamics (how occupants move within and between spaces) [54].

Preliminary investigations as to how such a model could ultimately be used to inform daylighting design through a goal-based approach, as conceptually illustrated in Figure 8, fourth row and further discussed in $[19,54]$, led to the need for a different simulation workflow for non-visual effects, where the absolute stimulus (here ocular light exposure, e.g. calculated as illuminance on a vertical plane) first has to be converted into a relative human response based on the model's outputs [19,53] then, in a second phase, gauged in terms of desirability in the considered context of specific building use and occupant profile (also influenced, e.g., by their sleep-wake cycle). Ultimately, the objective is to base the framework on performance goals that could indicate how appropriate (i.e. conducive to "health") the lighting pattern that the occupants are likely to be exposed to within that space (especially for healthcare or high-vigilance environments for instance) will be over the day and year.

\subsubsection{Delightful lighting}

Daylight has a fundamental role in generating dynamic visual interest in a space, which in many cases makes it a tangible - yet not always explicitated - guiding factor for design. To provide effective support regarding perceptual - i.e. often inherently emotional - aspects, we must seek to interpret subjective contrast and variability perception as design factors without objectifying them.

Two metrics, introduced in $[19,22,81]$, have been developed as a proof-of-concept: one expresses how "cumulative contrast" is distributed over space and time i.e. where (within a space) and when (over the day and/or year) we are likely to perceive the steepest gradients from dark to bright; the other one relates to the spatio-temporal variation of luminance as a "cumulative variability". Similarly, the latter evaluates where and when we will witness the most dramatic changes as time goes by in terms of bright versus dark areas. 
To evaluate daylight contrast or variability as defined above, a preliminary approach consists of starting from user-defined viewpoints that are used to frame the spatial area where contrast is analysed over time. To identify an upper bound for contrast, only clear sky conditions are used [81] and pixel value gradient from one pixel to the next (for contrast analyses) or change from one moment to the next (for variability) is calculated and cumulated over space - respectively over time - to obtain an overall "amount" of contrast or variability at any given moment of the year - respectively any given point in space (within the view frame).

Ultimately, the objective will be to develop a goal-based approach from a taxonomy of reference spaces, as illustrated in the last row of Figure 8 and further discussed in section 3.4.5.

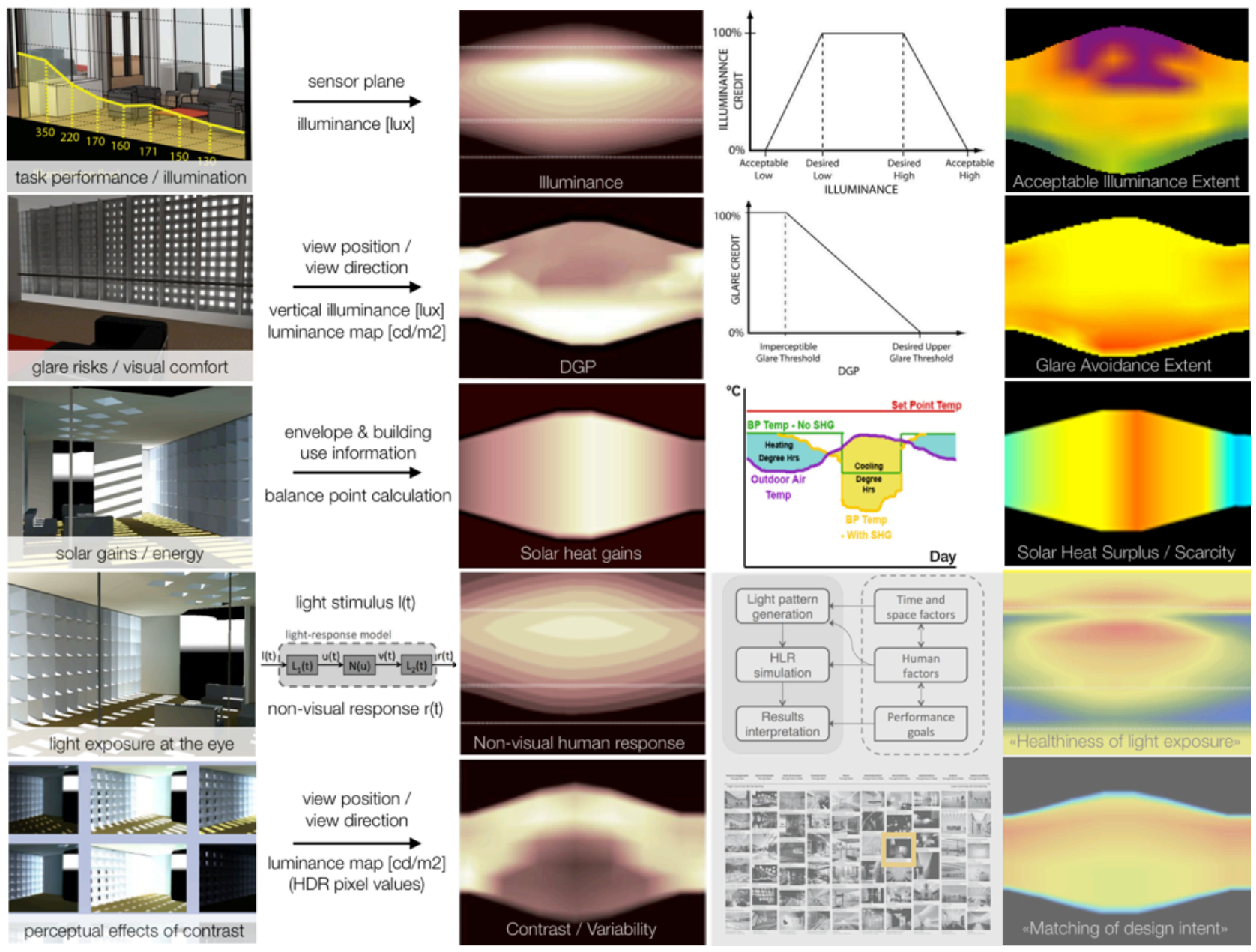

Fig. 8: Overview of performance analysis framework: five different perspectives (rows) regarding daylighting performance (left column) lead to five quantitative approaches to extract "absolute" performance (middle column) i.e. evaluated on a linear scale from low to high, used as inputs to threshold-based metrics enabling a goal-based visualization of performance (right column). Bottom-right modules (greyed) are work in progress.

\subsection{A unified framework for daylight dynamics}

To assist architectural designers in searching for "better" solutions to support a more flexible, user-centered approach, an annual daylighting simulation tool was developed in 2008 [89], called Lightsolve, meant to be used in the early design process [90] when façade and space details are still being defined. Lightsolve took a new perspective on daylighting analysis, focusing on the variation of daylight performance over the day and the year by combining temporal performance visualization with spatial renderings [18]. This simulation method included an expert system to support a guided search process and differed from previous approaches $[96,113]$ in that it allowed a comprehensive understanding of daylighting and offered user interactivity regarding design choices [88]. One of the underlying principles in how daylighting performance is 
evaluated in Lightsolve is to make the results specific to the user's own performance objectives and to his or her chosen areas of interest within the considered design project, hence making it an intriguing platform in which to integrate diverse aspects of performance. On the other hand, it combines a synthetic perspective of full-year data with a visual impression of what the space looks like over time.

A prototype interface is presented that offers an interactive, highly visual simulation environment in which to integrate these goal-based concepts. A preliminary workflow is proposed to integrate both conventional and unconventional - e.g. non-visual and perceptual - aspects of daylighting performance into an interactive tool specifically developed for early stage, full year, climate-based daylighting design support.

The following sections describe the goal-based simulation and visualization framework that enables the five very different perspectives described in section 3.3 to be considered in a unified and interactive way, according to a consistent color scale so as to compare "success" of highly dissimilar performance criteria. This study aims to demonstrate the potential of such a platform to embed the diversity of performance criteria relevant to daylighting while keeping a consistent and intuitive format across the board, that encompasses spatial as well as temporal properties of daylight. First the structure and interface of the platform itself is introduced, then its "double duality" is discussed with a spatial representation alongside a temporal one, and with performance evaluation being displayed both as absolute values and as how closely they fulfil user-defined performance goals, using an original color scale. Finally, a short introduction to ongoing work regarding the development of an iterative expert system is provided and two concrete examples of how such a framework can address non-conventional performance perspectives are presented.

\subsubsection{Revised structure of the Lightsolve simulation platform}

Initially developed as a simulation platform allowing fast annual renderings displayed simultaneously with a time-mapped visualization of daylighting performance $[18,20,90]$ and implemented as a SketchUp plugin, Lightsolve has recently been reprogrammed entirely [19]. Its overall principle [89] - and the general layout of the user interface (early prototype shown in Figure 9), with goal-based temporal maps displayed alongside interactive renderings [90], were maintained. Such temporal maps - with days of the year on the x-axis and time of day on the y-axis - exhibit how closely user-defined goals are fulfilled over the year using a triangular color scale [18].

The fundamental changes compared to the original version of Lightsolve relate to the new ray-tracing engines used and a completely new software and display structure. The main goal was to make the tool much more responsive and reliable and a specific set of tools and libraries was thus selected for this new structure to rely upon:

- for quantitative analyses, Lightsolve relies on the ubiquitous and extensively validated Radiance program [114] for illuminance calculations (that the visual task, glare and healthy lighting modules all use as inputs) and for luminance distribution analyses (necessary for perceptual lighting analyses and also glare evaluation)

- for qualitative analyses (i.e. mainly the visualization of renderings), a hardware-driven, GPU-based renderer (Nvidia Optix Application Acceleration Engine) has been implemented in the user interface to generate live renderings as the user explores the model and the lighting conditions: OptiX here acts as a real-time ray-tracing tool for ensuring high responsiveness and interactivity in the 3D rendering [115]

- $\mathrm{C}++$ is used as the programming language using GCC (GNU Compiler Collection) for Mac OSX or MinGW (Minimalist GNU) for Windows systems 

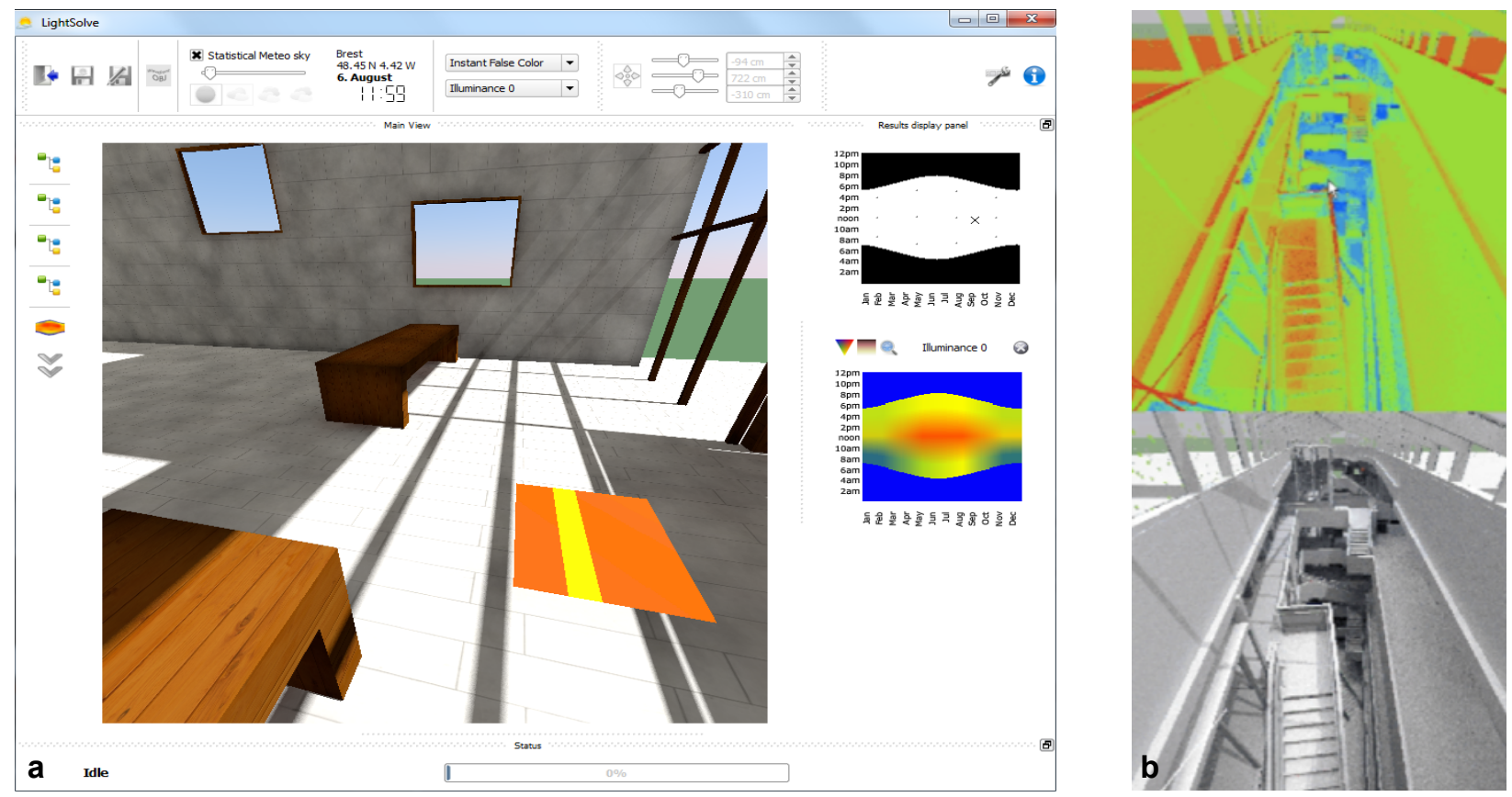

Fig. 9: Early prototype of the new Lightsolve version. a) User interface including goal-based analysis on user-defined sensor plane b) pixel-level illuminance map of atrium rendering: grey scale (bottom) versus false-color (top)

Currently, the Lightsolve structure is organized such that the core engine calculates all the key lighting variables (especially illuminance and luminance distributions on/ from selected surfaces/viewpoints) over the year, that are then used as inputs to individual modules, each corresponding to a given daylighting performance perspective or, rather, to its associated metric(s). In the case of the fifth considered perspective - perceptual daylight - two distinct modules had to be created to address contrast on the one hand and variability on the other hand. Based on these input data, each module computes the performance criteria associated to its metric, as described above. Results are fed back to the core engine for data display. Since the outputs strongly differ from one module to the other, the core engine automatically adapts the visualization according to the considered performance perspective.

The functionalities of the application are based on modules (one module per performance perspective or metric, broadly speaking) and are designed to allow both local and remote development [19]. These independent modules are detached from the core application to allow high flexibility in the development and later integration of new modules in the future. Two types of modular units can be distinguished: LightSolve Modules which are processing units with illuminance values as input, and LightSolve Services which are processing units with more complex inputs (Expert System, etc.). Only the latter can access and change whole scenes (mesh, position and point of view), start lighting simulation computing or reprocess modules. The Raytracing Engine and the Lighting Simulation Engine are also independent and detached from the core to allow future component replacements. Unlike LightSolve Services or Modules, the Raytracing and Lighting Simulation Engines are unique objects with precise goals and specific interfaces.

\subsubsection{Goal-based color scale}

The triangular color scale introduced in [18] and reproduced in Figure 10 is ideally suited to visualize the five daylighting performance perspectives within a consistent format, such that any outcome for any of their metrics can be

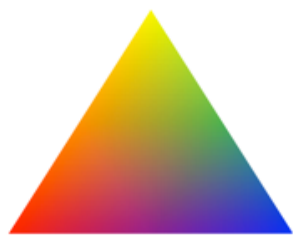

Fig. 10: Triangular color scale: yellow is $100 \%$ achievement of goals, red is $190 \%$ too high, 
visualized as a single graph. Combined with the temporal map format $[18,20]$ - with days of the year plotted along the $x$-axis and time of day along the $y$-axis such as in Figure 8 middle and right columns) -, three outcomes are possible for a single point study: either the resulting data falls within the desired range, or it exceeds the maximum, or it does not reach the minimum. For a multiple point analysis allowing for buffer intervals [18], any color combination could emerge (e.g. purple as in Figure 8 top right for a combination of too high (sunspots) and too low (overall too dim) illuminance levels within a given area of interest). By displaying goal compliance (Figure 8 right) instead of absolute response (Figure 8 middle), both the 'successfulness' of a design and the tradeoffs between dissimilar metrics become intuitive to understand, and harmful effects can easily be differentiated from beneficial ones.

\subsubsection{Visualization 'quartet'}

The Lightsolve interface and visualization framework reveals annual, seasonal and daily performance thanks to its time-based focus combined with a simultaneous visualization of spatial renderings [90]:

- time-varied performance (Fig. 11, left) is represented in the form of temporal maps over which a cursor (cross) can be moved to select a given moment over the year

- renderings (Fig. 11, right) are associated to that specific moment, where the spatial distribution of a considered metric's values (e.g. illuminance in the example shown in Figure 11) can be visualized in false-color on user-defined sensors (areas of interest).

Weather conditions are accounted for thanks to the climate-based time segmentation method described in [20] and renderings can be associated to either the dominant sky type for that period (clear, clear-turbid, intermediate or overcast) or to one chosen by the user.

As a second duality, these two representations of performance (temporal versus spatial) can each be displayed on either an "absolute" or a "goal-based" scale, leading to a visualization foursome or 'quartet' illustrated in Figure 11 for a West-facing room with a fully glazed façade, located at $41^{\circ} \mathrm{N}$ latitude:

- the so-called "absolute" scale (Fig. 11a) is based on a linear gradient from dark (low) to bright (high): it is a straightforward visualization of performance displaying the respective metric's average value over a chosen area of interest (e.g. illuminance over workplane like in Fig. 11a - other
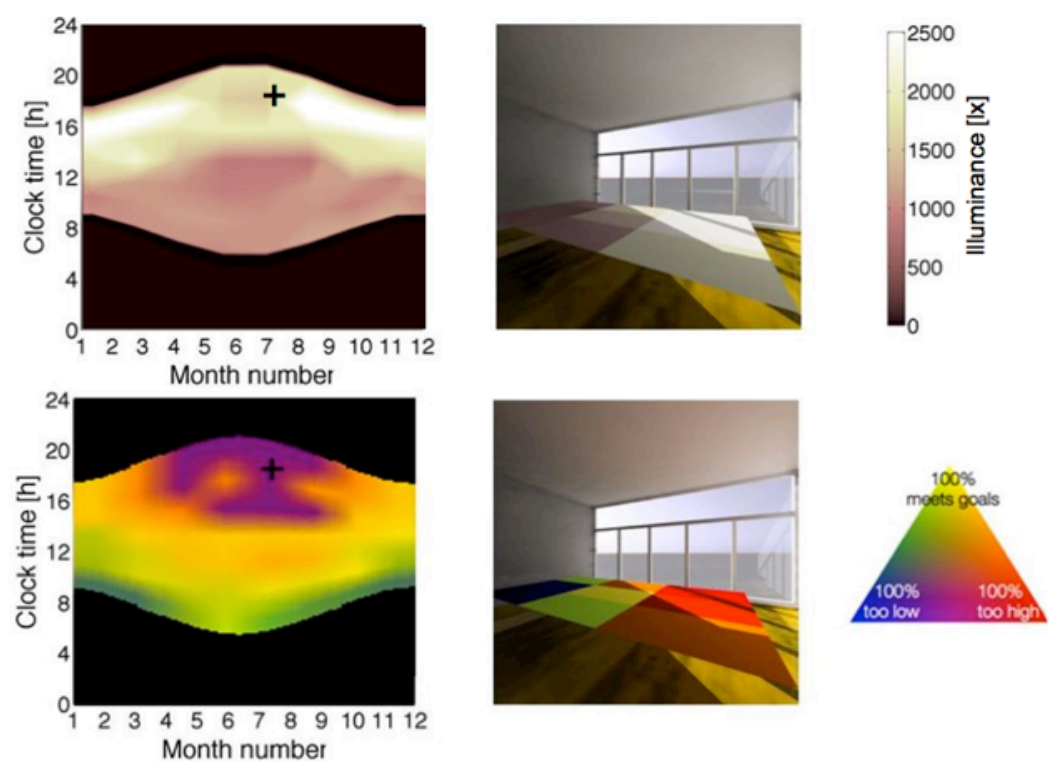

Fig. 11: Time-based illuminance analysis (left) with associated rendering at given moment (right and cursor) on an absolute (top) and goal-based scale (bottom) quantities could be displayed similarly, like average DGP, spatial contrast etc). It is important to note that the "absolute" scale can sometimes represent a relative response such as in the "healthy lighting" model.

- the "goal-based" scale (Figure 11b) is based on the triangular scheme described above (Fig. 10) and introduced in [18]: it represents how closely prescribed goals are met and translates absolute, time-based performance into a relative, goal-based scale (e.g. into Acceptable illuminance Extent (AIE, cf. section 
3.3.1) like in Fig. 11b, or Glare Avoidance Extent (GAE, cf. section 3.3.2) etc).

Fig. 11a is quite easy to interpret: considering a sensor plane covering almost the entire space at workplane height (Fig. 11a, right) and given the West-orientation of the space, average illuminance over the sensor area (left) will be higher in the afternoon all year long but even more so in the winter (unless the local climate has particularly cloudy winters). As far as spatial illuminance distribution is concerned for the selected moment under clear sky, illuminance will of course be highest on the sun spots (> 2500 lux) that are closest to the window, and lowest in the back corners ( $<500$ lux). Both temporal (climate-based maps) and spatial data (false-colored renderings) are based on the same color scale, provided on the left.

To obtain Fig. 11b, goals must be set: in this example, the targeted desired illuminance levels on the measurement area were between 800 and 1200 lux and the extreme acceptable values were 500 and 2000 lux. The obtained goal-based representation over a full year (as AIE values [\%]) here indicates that the objective is generally reached (dominating yellow), except during afternoons during the summer period, where objectives are not met because of simultaneously too high and too low illuminance values (depicted in purple). Confronting spatial and temporal information, one can easily explain the purple spots by the falsecolor rendering (Fig. 11b, right), with locations too dark (in blue) and others too bright (in red). More specifically, the sensor areas which receive direct sun rays are above the goal (red when higher than 2000 lux and in a variable orange when between 1200 and 2000 lux). Parts nearer to the left wall are either in blue (illuminances lower than 500 lux) or variable green (illuminances between 500 and 800 lux).

In terms of user experience, an exploration over time (i.e. by moving the cursor over a temporal map) gets translated into new renderings on the fly, and new view framings are shown as one moves within the rendered model. As each module delivers its output data to the core engine, the switching from absolute (Fig. 8, middle column) to goal-based (Fig. 8, right column) scale, from one moment to another (position on temporal map or user-defined choice of moment) and/or from one performance perspective or metric to the other (rows in Fig. 8) is almost instantaneous, providing the user with a dynamic framework to visualize and understand daylighting performance. The resulting "double combination" of absolute vs. goal-based and time-based vs. spatial visualization makes the performance analysis particularly interactive and - as previous studies have shown for the goal-based scale [90] - intuitive to the user.

\subsubsection{Guided search}

Ultimately, Lightsolve is meant to offer pro-active guidance regarding design decisions to the user / designer, based on how closely the considered design matches user-defined performance goals.

The capability to act as a "virtual consultant" by offering a feedback loop had been implemented in the original version of Lightsolve $[88,90]$. The expert system developed for that purpose was highly innovative from many aspects, especially its iterative approach allowing for a high educational potential [90] combined with the generation of a knowledge-base to rank design decisions based on their likelihood to result in an increased performance (with respect to user-defined goals) [87]. This initial expert system was, however, only applicable to illuminance and glare evaluation, and had important restrictions on orientation (façades facing cardinal directions) and geometry (square angles for all corners), which are amongst the limitations that the future expert system should be able to overcome.

As a prospective outlook to a more robust expert system, some preliminary concepts can be drawn and would require further work to be tested and implemented. It could be interesting e.g. to build a shareable knowledge-base of design effects, where performance impacts can be stored and where model similarities are evaluated to predict effects on performance, similarly to [116]. The idea would be to integrate user inputs 
through an immersive interface that would include a case-based 'Machine Learning' structure to collect and later match user inputs, and a 'Clustering' engine for design changes classification into representative groups [117]. This should lead to an iterative adoption of design changes, similarly to the original system but with a more efficient balancing of requests to the knowledge-base and to the calculation engine to provide information gain, drawing inspiration from information theory and market research [118].

Towards this end, we would have to maintain the ill-definition inherent to the creative design process [83] and build a dialog with the user respectful of implicit design intents (Figure 12), that avoids splitting the problem into smaller or alternative well-defined ones [85]. The ambition of such a system would thus be to emphasize educational potential around a goalbased structure where performance objectives can be implicit (subjective, e.g. preference) or explicit (objective, e.g. relative to prescribed thresholds), and to offer an iterative, open-ended platform to engage the user in the exploration of an inherently incomplete

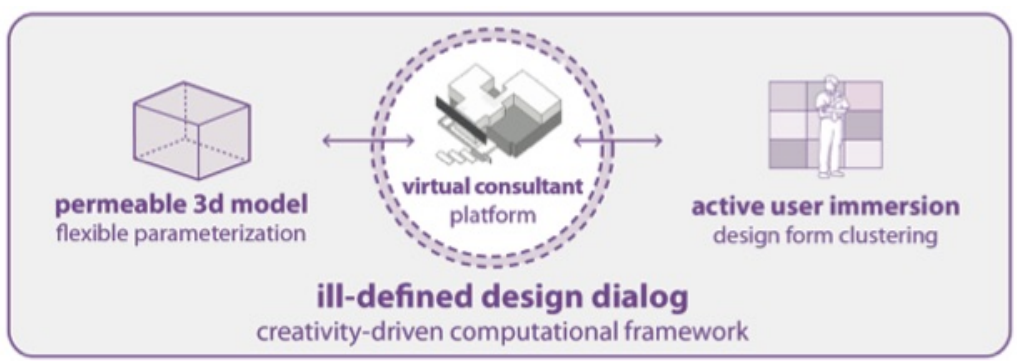

Fig. 12: Prospective research directions that could be explored to embed ill-definition in a decision supporting framework problem-space.

\subsubsection{Application to two unconventional interpretations of daylighting performance}

This section presents a proof-of-concept for the above-described goal-based simulation structure that could offer design support for daylighting performance aspects beyond conventional ones (such as illumination, glare or solar gains), with a focus on health, drawing from the latest findings in photobiology in terms of effects on sleep, health and well-being, and on the perceptual qualities of daylight through a dynamic analysis of spatial contrast and its variability over time.

\section{Addressing non-visual effects}

A dynamic model of the non-visual light-response relationship is being developed that combines two temporal integration modules and a nonlinear response function [19,53,55], whose application to an actual design exercise has been discussed previously in [54]. What we propose here is test how effectively its (future) outcomes can be formatted within the context provided by Lightsolve, i.e. as goal-based, time-driven performance maps. Linking the model to a lighting simulation process is indeed important, because the ultimate goal is to develop a new type of lighting design support.

As an example of a preliminary format where goal-fulfilment in this human-centered context could be visualized, the same West-facing room used previously (Fig. 11) was modelled with a window facing West. Daylight exposure (vertical illuminance) for someone who would stand in the middle of the room and stare at the window all year long is provided in Figure 13a, based on Radiance calculations and on Daysim's daylight coefficient method. Figure 13b shows the estimated nonvisual response once our model is applied and where one can clearly observe the effect of our non-visual system's sluggishness (response delayed compared to exposure).

To adopt a goal-based perspective, since the non-visual system adapts to changes in light intensity and spectral composition over a much longer time period than the
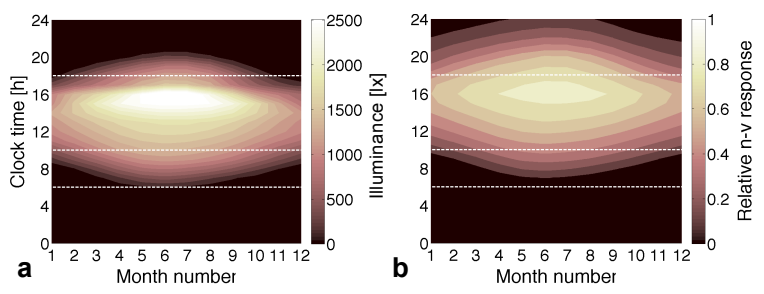

Fig. 13: Illuminance (a) vs. relative non-visual response as predicted by our proposed light-response model (b) 
visual system, we cannot apply instantaneous performance criteria. If a non-visual response is categorized as "bad" after evaluating Fig. 13b's results against prescribed goals, it is far from trivial to trace the response back to a specific period of time over the day when light exposure was too low or too high (Fig. 13a). The main difficulty in tracing back the cause is that there exists not only one but an infinite number of light patterns that may induce the same non-visual effects. In order to achieve this, it will be necessary, in the future, to develop a search algorithm that will look for a set of light patterns that best fit the performance criteria.
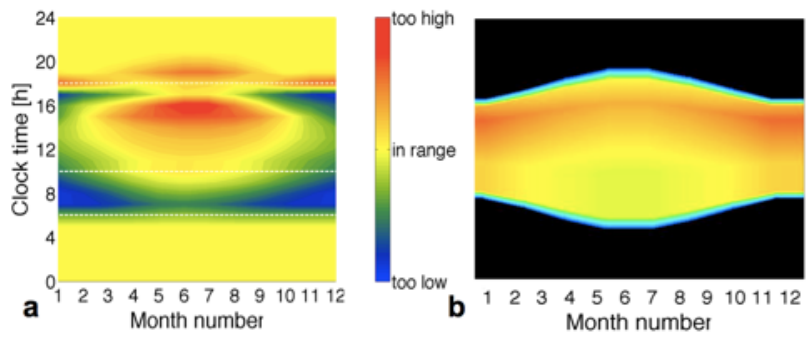

Fig. 14: Conceptual maps for goal-based performance representation of a) non-visual effects and b) contrast

Conceptually, such an approach would lead to outcomes that may look like the one displayed in Fig. 14a. The figure shows periods of time when light exposure should be avoided where it could have negative influences on circadian rhythms (red-orange) and when more light could have beneficial effects in terms of alertness and productivity (blue-green). The horizontal lines in Figs 13 and 14a mark three time periods differing from one another in terms of their phase-shifting effects: light exposure in the late day/early night typically causes a phase delay, whereas light in the late night/early day will phase advance the clock; light exposure in the late morning and early to mid-afternoon usually has little impact on phase shifting but might influence alertness and other direct effects. A goal-based performance criterion is assigned to each period to account for timing of light exposure relating to the circadian clock.

\section{Incorporating perceptual aspects}

Based on the medium of photographs and rendered scenes, which architects use readily within the practice of design, a dual approach for perceptual daylight analysis in the form of complementary metrics for contrast and variability has been introduced in $[22,81]$. Its implementation as a Lightsolve metric is discussed below and its conceptual interpretation from a goalbased perspective is introduced as a work in progress.

Spatial Contrast estimates the spatial distribution of contrast across a selected view at a given moment; its annual dynamic variability can be represented using an absolute-scale temporal map in Lightsolve (Fig. 15a, left), which shows when that contrast will change
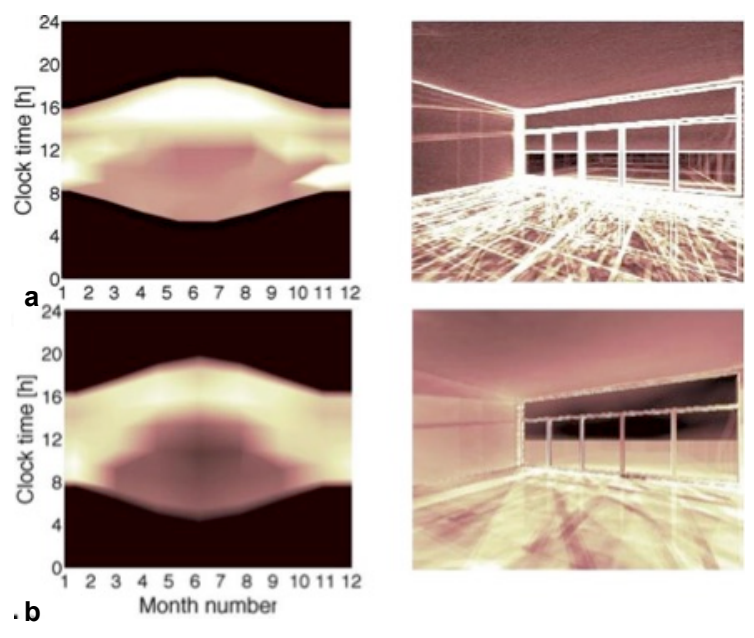

Fig. 15: Year-cumulative (a) perceptual contrast and (b) luminance variability for framed view as 'absolute' values (left: temporal maps) vs. space (right: spatial throughout the day and year as a result of sunlight dynamics (all afternoons and mornings in the winter in this example). To complement this time-based information, instantaneous false-color renderings of spatial contrast can be produced for every moment and displayed simultaneously in the Lightsolve interface to show where (i.e. in which portions of the view) most spatial contrast will be experienced. But one can also display cumulative annual contrast renderings (Fig. 15a, right) to evaluate spatial contrast when considering the entire year (in the example chosen, the annual contrast is clearly highest over the floor plane).

Luminance variability throughout a selected view, on the other hand, calculates the absolute difference in pixel values between daily and seasonal instances. Similarly to contrast, one can generate instanteaneous (from one time-step to the next) but also cumulative (annual) luminance variability (Fig. 15b, right) to highlight which areas experience the most frequent change over the year (here again on the floor and to 
some extent on the wall). The associated temporal map (Fig. 15b, left), shows when the most variability occurs through the day and year (afternoons, and throughout the day in the winter months).

To provide support in manipulating parameters to achieve a desired effect regarding perceptual daylight performance (i.e. take a goal-based perspective), the 'non-absoluteness' of performance takes its full meaning more than ever: there is no objectively "good" or "bad" performance (except in terms of norms or comfort requirements), what counts is the designer's intent. In the design support framework that Lightsolve offers, reference spaces, such as those found in the taxonomy generated to develop the metrics [81] and represented in Fig. 16a (with most extreme contrast and/or variability spaces on the left of the gradient and the more uniform / stable ones on the right), can be used. They should represent expressions of contrast and variability patterns architects can select as desirable luminous characters, regardless of their idio-syncratic interpretation of the quality of each space. As such, they can thus serve as a "delight target" database (i.e. a set of freely-interpreted effects ranging from low to high contrast and/or variability). Simplified 3D models, associated to each type of lighting composition (Fig. 16b) and expressing similar gradients and compositions of contrast or variability as the corresponding spaces but with a more abstracted level of detail, can then serve as a support for time-lapse renderings. From the latter, our metrics, used as indicators of performance, can be calculated and graphed and used as a target for a guided search, informing the design process without constraining it.

The theoretical example shown in Fig. 14b shows how we might represent the degree of annual spatial contrast in terms of goal-based targets established by the designer. Using the same west-facing space as an example - corresponding to type 5 in Fig. 16b -, let's assume the designer had in mind to achieve high contrast to stimulate visual interest but keep it within a certain threshold (closer to what a space corresponding to type 4 with a higher shading resolution (here horizontal louvers in the lower portion of the window) would generate e.g.). A framework like Lightsolve would then generate a goal-based temporal map conceptually similar to Fig. 14b: it would exhibit "too high" spatial contrast (compared to target goals) in the winter (especially afternoons) - leading to red or orange - and acceptable spatial contrast in the mornings and most of the summer days - leading to yellow. Iterating from an initial scenario (say the one leading to the analysis shown in Fig. 15a), designers would then be able to investigate changes within their design model that would more closely meet their design goals (which would lead to a more 'yellow' map in Fig. 14b).

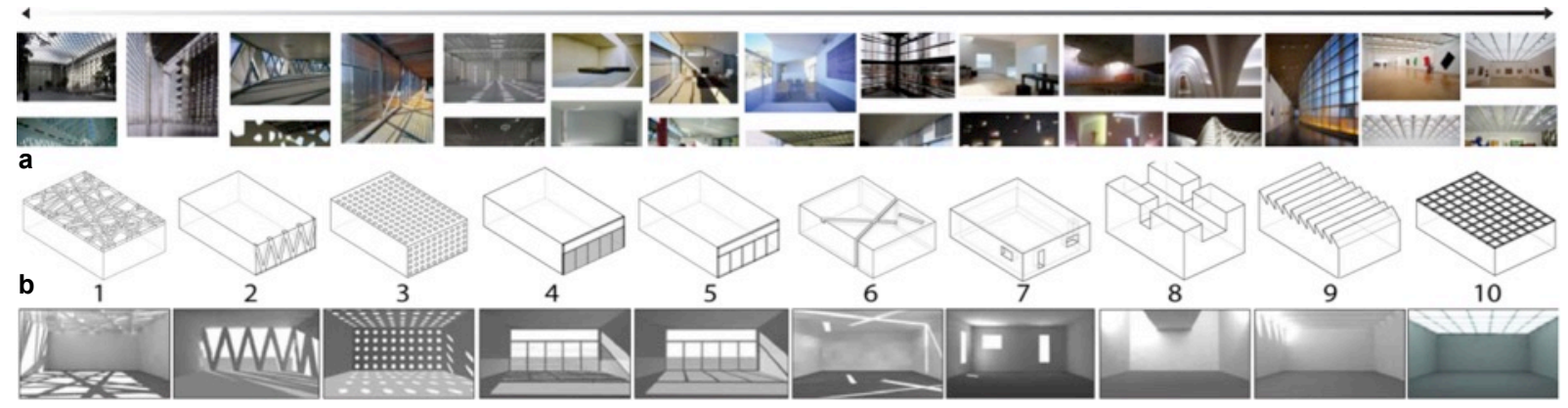

Fig.16: Taxonomy of contrast / variability gradients in a) architectural spaces and b) typological matrix

As illustrated by this conceptual analysis, performance can be understood, even for somewhat subjective, intent-driven metrics expressing contrast or variability, by how closely a desired effect will be matched, i.e. how similar the metrics' values are between the desired effect (target, here associated with an image) and the considered one. Such an approach, which is currently under development, will ultimately allow objective comparisons between spaces while keeping the perceptual aspects of light intact: the designer will still be able to freely interpret light quality with his/her own sensitivity. 


\section{Conclusion}

Daylight is a desirable architectural component that satisfies both visual and psychological needs of occupants while it is derived from variable and non-deterministic environmental parameters. This paper discusses emerging research fields at the interface between daylighting design and more fundamental domains related to human interactions with their environment, thereby strengthening connections between fields as far from design as neuroscience and psychophysics, brought together around a human-centered perspective. Exciting research pathways, such as pertaining to non-visual effects of light, to the gaze dynamics relevant in work environments and the perceptual qualities of daylight regarding contrast, are being explored. These core qualitative and physiological aspects, that are inherent to daylight performance yet are not as easily associated with absolute thresholds of "good" versus "bad", must be put back into the design realm. They indeed bring the promise of novel findings both on how we design buildings and how the design process can interface with fundamental discoveries about humans and their behavior. We have access to the essential ingredients of human-responsive design, now we need to cook.

Daylighting requires us to deal with very different quantities all relevant to 'good' daylighting performance (illumination potential, glare risks, aesthetics, overheating risks, physiological effects of light...), which reveal themselves complementary drivers for design decisions. The objective is also to find the way they can interactively, dynamically and effectively fuel the ill-defined, creative design process.

Towards this end, the paper introduces a goal-based approach, adopted by the Lightsolve simulation framework, to bring together physical, physiological and perceptual aspects of daylight around the temporal variability of their effects, evaluated on a relative basis for five performance perspectives within a unique, intuitive and visual format. The objective is to support early stage design regarding both conventional aspects of daylight performance such as workplane illuminance, glare and associated solar gains, and unconventional ones such as perceptual or non-visual effects of daylight, all considered in combination within a unified framework of analysis. The results show the potential of such visualization formats to express annual daylighting performance consistently even for radically different aspects for which time-dependency represents the most essential aspect of performance. Goal-based color scales are particularly powerful in proposing a unified framework to describe performance in terms of how closely prescribed goals are met, and thus assessing how "successful" a design option is (especially in relative terms) from these different perspectives. The workflow, competences and design stage best supported by this framework are expected to be the same or very similar to those applicable for the user studies described in [90], though a dedicated design-driven validation study would be needed to specifically evaluate its ability to support early-stage design decisions regarding the many declinations - whether conventional or unconventional - of daylighting performance.

Inter-disciplinary research directions are needed to build the basis on which human-responsive daylighting design can be promoted, fully embracing its complexity and dynamics. New research prospects are being opened as to how we could empower the designer with computation and further investigate the relationships between occupants and a built environment with a focus on their dynamics, driven by fundamental questions on how we interact with our environment: how sensitive are we to our built environment? are some design elements significantly more impactful on how healthy one might be in a space, or how much one enjoys it? is there a significant difference between objective and perceived impact of a design change? does the advent of computing technologies necessarily change the role of the architect? 
Looking for answers to these questions will foster a re-thinking of the integration of technology both in the design process and in actual building systems. And beyond searching for technological solutions, increasing building performance in its broadest, most multifaceted sense, should become an inherent part of the design process that focuses on the reason we build buildings in the first place: to shelter complex, diverse occupants.

\section{Acknowledgements}

This work was supported by the Ecole Polytechnique Fédérale de Lausanne (EPFL).

I would like to thank my $\mathrm{PhD}$ students for their contributions to the underlying concepts and models described in this paper, and especially Mandana Sarey Khanie, Maria Lovisa Amundadottir and Siobhan Rockcastle, whose ongoing thesis work forms the basis of some of the discussed research developments, which will be partially funded, respectively, by the Swiss National Science Foundation for the first two and the Velux Foundation for the third after 2015. Siobhan also significantly contributed to the creation of many of the graphics contained in this paper, especially in section 2.

I would also like to thank Lorenzo Cantelli who has been developing the new Lightsolve prototype, with, in its initial phase of re-development, Dr. Antoine Guillemin's help and supervision.

\section{References}

[1] L Pérez-Lombard, J Ortiz, C Pout, A review on buildings energy consumption information, Energy and Buildings 40(3), 394-398, 2008

[2] P Ihm, A Nemri, M Krarti, Estimation of lighting energy savings from daylighting. Building and Environment, 44(3), 509-514, 2009

[3] International Energy Agency, Testing and Validation of Building Energy Simulation Tools, Tech. Rep. SHC Task 34, 2008

[4] C Reinhart, J Wienold, The daylighting dashboard: a simulation-based design analysis for daylit spaces, Build. \& Env 46(2), 2011

[5] A Galasiu, J Veitch, Occupant preferences and satisfaction [...] in daylit offices: a literature review, Ener. \& Build. 38(7), 2006

[6] P Correia da Silva, V Leal, M Andersen, Influence of shading control patterns on the energy assessment of office spaces, Energy \& Buildings 50, 2012

[7] J Wienold, J Christoffersen, Evaluation methods and development of a new glare prediction model [...], Ener. \& Build. 38(7), 2006

[8] MC Dubois, Shading devices and daylight quality: an evaluation based on simple performance indicators, Lighting Research and Technology 35(1), 2003

[9] CF Reinhart, K Voss, Monitoring manual control of electric lighting and blinds, Lighting Research and Technol. 35(3), 2003

[10] J Wienold, Dynamic daylight glare evaluation, In Proc. of International IBPSA Conference, 2009

[11] J Veitch, Psychological processes influencing lighting, J of the Illuminating Eng. Soc 30, 2001

[12] PR Boyce, Human factors in lighting, Taylor \& Francis, 2004

[13] L Edwards, PA Torcellini, A literature review of the effects of natural light on building occupants, NREL, 2002

[14] L Heschong, Daylighting and human performance, ASHRAE J 44(6), 2002

[15] M Rea (Ed.), The IESNA Lighting Handbook, The Illuminating Engineering Society, 9th Edition, 2000

[16] GR Newsham, et al, Lighting quality recommendations for VDT offices: a new method of derivation, Lighting Research and Technology 33, 2001

[17] Mardaljevic J, Heschong L, Lee E. 2009. Daylight metrics and energy savings, Lighting Research and Technology 41(3): 261-283

[18] S Kleindienst, M Andersen, Comprehensive Annual Daylight Design through a Goal-Based Approach, Building Research \& Information 40(2), 2012

[19] M Andersen, A Guillemin, ML Amundadottir, S Rockcastle, Beyond illumination: An interactive simulation framework for non-visual and perceptual aspects of daylighting performance, In Proceedings IBPSA 2013, Chambéry, France, Aug 26-30, 2013

[20] S Kleindienst. M. Bodart, M. Andersen, Graphical Representation of Climate-Based Daylight Performance to Support Architectural Design, LEUKOS 5(1), 2008

[21] GR Newsham, C Richardson, C Blanchet, JAVeitch, Lighting Quality Research Using Rendered Images of Offices. Light Res Techn 37 (2): 93-115, 2005

[22] S Rockcastle, M Andersen, Dynamic Annual Metrics for Contrast in Daylit Architecture, Proc. SimAUD 2012, Orlando FL, 2012

[23] S Dave, M Andersen, A Comprehensive Approach for Determining Performance Metrics for Complex Fenestration Systems, Winter 2012 ASHRAE Transactions 118 (1): 392, Chicago, Jan 2012

[24] S Lockley, Circadian rhythms: Influence of light in humans, Encyclopedia of Neuroscience (5), 2009

[25] SW Lockley et al, Visual impairment and circadian rhythm disorders [Review]. Dialogues in Clinical Neuroscience 9(3), 2007

[26] R Stevens, M Rea, Light in the Built Environment: Potential role of Circadian Disruption [...], Cancer Causes and Contr 12, 2001

[27] AU Viola et al., Blue-enriched white light in the workplace improves self-reported alertness, performance and sleep quality. Scandinavian J. of work, environment \& health, 34(4):297-306, 2008

[28] K Beauchemin, P Hays, Dying in the dark: sunshine, gender and outcomes in myocardial infarction, J Royal Soc Med 91(7), 1998

[29] C Cajochen et al., Dose-response relationship for light intensity and ocular and electroencephalographic correlates of human 
alertness. Behavioral Brain Research 115(1):75-83, 2000

[30] JM Zeitzer, D-J Dijk, RE Kronauer, EN Brown, CA Czeisler, Sensitivity of the human circadian pacemaker to nocturnal light: melatonin phase resetting and suppression. J Physiol 526(3), 2000

[31] D Berson et al, Phototransduction by retinal ganglion cells that set the circadian clock, Science 295, 2002

[32] Webb A. 2006. Considerations for lighting in the built environment: Non-visual effects of light. Energy and Buildings, 38(7):721-727

[33] CS Pechacek, M Andersen, SW Lockley, Preliminary method for prospective analysis of the circadian efficacy of (day)light with applications to healthcare architecture, LEUKOS 5(1), 2008

[34] C Czeisler, K Wright, Influence of light on circadian rhythmicity, Neurobiology of sleep and circadian rhythms, M Dekker, 1999

[35] J Phipps-Nelson et al, Daytime exposure to bright light, as compared to dim light, decreases sleepiness [...], Sleep 26(6), 2003

[36] M Rueger et al, Time-of-day-dependent effects of bright light exposure [...], American j. of physiol. 290(5), 2006

[37] Chang A-MM, et al. 2012. Human responses to bright light of different durations. The Journal of physiology, 590(13):3103-3112

[38] C Gronfier et al, Efficacy of a single sequence of intermittent bright light pulses [...], Am J Physiol Endocrin Metab 287(1), 2004

[39] DW Rimmer et al., Dynamic resetting of the human circadian pacemaker [...], Am. J. of Physiol. 279(5), 2000

[40] AMM Chang et al., The human circadian system adapts to prior photic history, The Journal of physiology 589(5), 2011

[41] K Smith et al, Adaptation of Human Pineal Melatonin Suppression by Recent Photic History, J of Clin Endoc Metab 89(7), 2004

[42] SW Lockley et al, Short-wavelength sensitivity for the direct effects of light on alertness, vigilance, and [...], Sleep 29(2), 2006

[43] M Münch et al., Effects of prior light exposure on early evening performance, subjective sleepiness, and hormonal secretion. Behavioral neuroscience, 126(1):196-203, 2012

[44] GC Brainard et al., Action Spectrum for Melatonin Regulation in Humans [...], The J. of Neuroscience 21(16), 2001

[45] JJ Gooley, et al., Spectral Responses of the Human Circadian System Depend on the Irradiance and Duration of Exposure to Light. Science Translational Medicine, 2(31ra33), 2010

[46] MS Rea et al., Modeling the spectral sensitivity of the human circadian system, Light Res Technol, 2011

[47] M Rea, M Figueiro, A Bierman, J Bullough, Circadian light, Journal of Circadian Rhythms 8 (1), 2010

[48] H Arsenault et al, Effects of glazing colour types on daylight quality, arousal and switch-on patterns [...], Glass Perf. Days, 2011

[49] RP Leslie et al., Conceptual design metrics for daylighting, Lighting Research and Technology, 2011

[50] RE Kronauer et al, Quantifying human circadian pacemaker response to [...] light stimuli [...], J of Biol Rhythms 14(6), 1999

[51] M Andersen, SJ Gochenour, SW Lockley, Modelling 'non-visual' effects of daylighting in a residential environment. Building and Environment, vol. 70, p. 138-149, 2013

[52] M Andersen, J Mardaljevic, SW Lockley, A Framework for Predicting Non-Visual Effects of Daylight: Part I - Photobiology-based model, Lighting Research \& Technology 44(1):37-53, 2012

[53] ML Amundadottir, M St Hilaire, SW Lockley, M Andersen, Modeling non-visual responses to light: unifying spectral sensitivity and temporal characteristics in a single model structure. CIE Conference, Paris, France, April 15-16, 2013

[54] ML Amundadottir, SW Lockley, M Andersen, Simulation-based evaluation of non-visual responses to light: proof-of-concept study of healthcare re-design, BS 2013, August 26-30, 2013 Chambéry, 2013

[55] ML Ámundadóttir, S. W. Lockley and M. Andersen. Integrating non-visual effects of light into lighting simulation: challenges ahead. LuxEuropa 2013: 12th European Lighting Conference, Krakow, Poland, September 17-19, 2013

[56] PR Boyce, Lighting research for interiors: the beginning of the end or the end of the beginning, Light Res Technol 36(4), 2004

[57] J Vos, Reflections on glare, Lighting research technol. 2, 2003

[58] I Ashdown, Sensitivity Analysis of Glare Rating Metrics, LEUKOS 2(2), 2005

[59] W Osterhaus, Discomfort glare assessment and prevention for daylight applications in office environments, Sol Ener 79(2), 2005

[60] W Kim et al, The position index of a glare source at the borderline between comfort and discomfort [...], Build. \& Env. 44, 2009

[61] J Jakubiec, C Reinhart, The adaptive zone - a concept for assessing discomfort glare [in] daylit spaces, Light Res Tech $44(2), 2012$

[62] AL Yarbus, Eye movements and vision, Plenum Press, New York, 1967

[63] W Einhäuser et al, Task-demands can immediately reverse the effects of sensory-driven saliency [...], J of Vision 8(2), 2008

[64] A Torralba et al, Contextual guidance of eye movements and attention in real-world scenes [...], Psychological Rev. 113, 2006

[65] R Krauzlis, Eye movements, In Fundamentals of Neuroscience (Squire L. et al, Eds.), 2008

[66] L Itti et al, A model of saliency-based visual attention for rapid scene analysis, IEEE Pattern Anal \& Machine Intell 20(11), 1998

[67] V Navalpakkam, C Koch, P Perona, Homo economicus in visual search, Journal of Vision 9(1), 2009

[68] MF Land, DN Lee, Where we look when we steer, Nature 369, 1994

[69] M Hayhoe, D Ballard, Eye movements in natural behavior, Trends in Cognitive Sciences 9, 2005

[70] MF Land, P McLeod, From eye movements to actions: How batsmen hit the ball, Nature Neuroscience 3, 2000

[71] M t'Hart et al, Gaze allocation in natural stimuli: comparing free exploration to head-fixed viewing conditions, Vis Cog 17(6), 2009

[72] S Hubalek, C Schierz, LichtBlick - photometrical situation and eye movements at VDU work places, Lux Europa conf, 2005

[73] M Sarey Khanie, S Mende, J Stoll, W Einhäuser, M Andersen, Investigation of gaze patterns in daylit workplaces: using eyetracking methods to objectify viei direction as a function of lighting conditions, In Proc CIE Conference, Paris, France, 2013

[74] M Sarey Khanie, M Andersen, B. M. 't Hart, J. Stoll and W. Einhäuser. Integration of Eye-tracking Methods in Visual Comfort Assessments. CISBAT 11 conference, Lausanne, Switzerland, September 14-15, 2011.

[75] M Sarey Khanie, J Stoll, S Mende, J Wienold, W Einhäuser et al. Uncovering relationships between view direction patterns and glare perception in a daylit workspace. LUXEUROPA, Krakow, Poland, September 17-19,2013

[76] E Schneider et al, EyeSeeCam: An Eye Movement-Driven Head Camera [...], Annals of NY Acad of Sciences 1164(1), 2009

[77] MA Steane, K Steemers, Environmental Diversity in Architecture, Spoon Press, New York, 2004

[78] D Cetegen, JA Veitch, GR Newsham, View Size and Office Illuminance Effects on Employee Satisfaction. Proc. Balkan Light, Ljubljana, 2008

[79] K Wymelenberg, M Inanici, A Study of Luminance Distribution Patterns and Occupant Preference in Daylit Offices, PLEA 2009, Quebec City, 2009

[80] K Parpairi et al., The Luminance Differences index: a new indicator of user preferences in daylit spaces, Lighting Research \& Technology 34, 2002

[81] SF Rockcastle, M Andersen, Measuring the Dynamics of Contrast \& Daylight Variability in Architecture: A Proof of Concept Methodology, Building and Environment, in press (DOI:10.1016/j.buildenv.2014.06.012)

[82] A Liljefors, Lighting and Color Terminology, CIE Discussion, Stockholm, 1997

[83] Y Kalay. Redening the role of computers in architecture: from [...] tools to knowledge-based design assistants, CAD 17(7), 1985 
[84] HA Simon, The structure of ill-structured problems, Artificial Intelligence 4, 1984

[85] W Zeiler, P Savanovic, E Quanjel. Design decision support for the conceptual phase of the design process, IASDR, 2007

[86] J Marks, et al, Design galleries: a general approach to setting parameters for computer graphics and animation, SIGGRAPH, 1997

[87] JML. Gagne, M Andersen, A daylighting knowledge-base for performance-driven facade design exploration, LEUKOS 8(2), 2011

[88] JML Gagne, M Andersen, LK Norford, An Interactive Expert System for Daylighting Design Exploration, Building and Environment 46(11), 2011

[89] M. Andersen, S. Kleindienst, L. Yi, J. Lee, M. Bodart, B. Cutler, An intuitive daylighting performance analysis and optimization approach, Building Research \& Information 36(6), 2008

[90] M Andersen, JML Gagne, S Kleindienst, Interactive expert support for early stage full-year daylighting design: a user's perspective on Lightsolve, Automation in Construction, vol 35, p. 338-352, 2013

[91] LG Caldas, LK Norford, A design optimization tool based on a genetic algorithm, Automation in Construction 11, 2002

[92] K Shea, R Aish, M Gourtovai. Towards integrated performance-driven generative design tools, Automation in Construction 14 , 2005

[93] JML Gagne, M Andersen, A Generative Façade Design Method Based on Daylighting Performance Goals, Journal of Building Performance Simulation 5(3), 2012

[94] A Yezioro, A knowledge based CAAD system for passive solar architecture, Renewable Energy 34(3), 2009

[95] T Jung, M Gross, E Do, Light pen: sketching light in 3D, Proc. of 10th CAAD Futures, 2003

[96] B Paule et al, DIAL+ a complete but simple suite of tools to optimize the global performance of buildings openings, CISBAT, 2011

[97] GG Wang, S Shan, Review of Metamodeling Techniques in Support of Engineering Design Optimization, J of Mech Design, 2006

[98] W Wang et al, Applying multi-objective genetic algorithms in green building design optimization, Build and Environ 40,2005

[99] R Choudhary, J Michalek, Design optimization in computer aided architectural design, Intern. Assoc. CAAD Res. in Asia, 2005

[100] R Oxman, Performative design: a performance-based model of digital architectural design, Planning and Design 36(6), 2009

[101] A Brintrup et al, An interactive genetic algorithm-based framework for qualitative criteria [...], Computers in Industry 58(3), 2007

[102] C Demers, Assessing light in architecture: a numerical procedure for qualitative and quantitative analysis, CIE conference, 2006

[103] H do Nascimento, P Eades, User hints: a framework for interactive optimization, Future Generation Computer Syst. 21(7), 2005

[104] G Luger, Artificial Intelligence: structures and strategies for complex problem solving, Addison-Wesley Longman, 2005

[105] D Anderson, et al, Human-guided simple search, Proc. 7th Nat. Conf. on Artificial Intelligence, 2000

[106] S Taras et al, An interactive multi-objective optimization [...] for sustainable design of bioprocesses, Comp \& Chem Eng 43, 2012

[107] H Takagi, Interactive evolutionary computation: Fusion of the capacities of EC optimization \& human evaluation, IEEE 89, 2001

[108] P Pu, D Lalanne, Design Visual Thinking Tools for Mixed Initiative Systems, Proc. Intelligent User Interfaces, ACM Press, 2002

[109] S Stevens et al, Virtual Pedagogical Agents as Aids for High School Physics Teachers, ICL2007 conference, 2007

[110] S Stevens et al, Coupling Conversational Interactivity with other Presentation Modes [...], Artif. Intelligence in Educ conf, 2009

[111] X Ge, SM Land, Scaffolding Students'Problem-Solving Processes in an III-Structured Task Using [...], ETR\&D 51(1), 2003

[112] M Andersen, A Guillemin, Daylight dynamics to guide early stage design: a user-driven goal-based approach to "good" lighting. Proc. PLEA 2013 conference, Munich, Germany, September 10-12, 2013

[113] C Ochoa, I Capeluto, Advice tool for early design stages of intelligent façades based on energy and visual comfort approach, Energy \& Buildings 41(5), 2009

[114] G Ward, RA Shakespeare, Rendering with Radiance - The Art and Science of Lighting Visualization, MK Publishers, 1998

[115] JD Nasman B Cutler B, Evaluation of a tangible interface for architectural daylighting analysis, i3D:207-ACM SIGGRAPH, 2011

[116] P-A Champin, ARDECO: an assistant for experience reuse in Computer Aided Design, Proc WS 5 of ICCBR'03, 2003

[117] A Machwe, Agency-based integration of aesthetic criteria within an interactive evolutionary design environment, Bristol IT, 2008

[118] JR Quinlan, Induction of Decision Trees, Machine Learning 1, 1986 